\title{
Nonprogression with avelumab treatment associated with gains in quality of life in metastatic Merkel cell carcinoma
}

\author{
Howard L Kaufman ${ }^{1}$, Matthias Hunger ${ }^{2}$, Meliessa Hennessy ${ }^{3}$, Michael Schlichting ${ }^{4}$ \& \\ Murtuza Bharmal*,4 \\ ${ }^{1}$ Massachusetts General Hospital, Boston, MA, USA \\ ${ }^{2}$ Mapi, Munich, Germany \\ ${ }^{3}$ EMD Serono, Boston, MA, USA \\ ${ }^{4}$ Merck KGaA, Darmstadt, Germany \\ * Author for correspondence: murtuza.bharmal@merckgroup.com
}

\begin{abstract}
Aim: To assess the association between tumor response and health-related quality of life (HRQoL) in patients with metastatic Merkel cell carcinoma treated with the anti-PD-L1 avelumab. Materials \& methods: Phase II single-arm trial (NCT02155647) data of 88 patients were analyzed. Correlations between percentage reduction in tumor size and change from baseline in Functional Assessment of Cancer Therapy General (FACT-G), FACT - Melanoma (FACT-M) and EuroQol-5 Dimension scores were calculated. HRQoL and utility by tumor response (per the Response Evaluation Criteria In Solid Tumors version 1.1) was estimated. Results: Tumor shrinkage correlated positively with patients' change from baseline in the FACT-M total (0.364 [95\% Cl: 0.050-0.607]) and subscale scores. Differences in HRQoL and utility between nonprogressive disease and progressive disease were clinically relevant. Conclusion: In patients with metastatic Merkel cell carcinoma, nonprogression during treatment with avelumab correlated with gains in HRQoL.
\end{abstract}

Trial registration: This study is a planned exploratory analysis conducted on data collected in Part A of the JAVELIN Merkel 200 trial. This trial was registered on 2 June 2014 with ClinicalTrials.gov as NCT02155647.

First draft submitted: 14 September 2017; Accepted for publication: 16 October 2017; Published online: 8 December 2017

Keywords: EQ-5D • FACT-M questionnaire • Merkel cell carcinoma • patient reported outcomes • quality of life • tumor progression

Merkel cell carcinoma (MCC) is a rare, very aggressive primary cutaneous carcinoma of the skin [1]. In Europe, a crude incidence rate of 0.19 per 100,000 between 2000 and 2007 was reported [2]. In the USA, the incidence rate reported in the most recent analysis of Surveillance, Epidemiology, and End Results data was 0.79 per 100,000 in 2011 [3]. The disease-associated mortality of MCC is estimated to be between 33 and 46\%, reflecting the aggressiveness of the tumor $[4,5]$.

Standard of care across all treatment guidelines is surgery in stages I-III of the disease. MCC is considered to be a chemotherapy-sensitive tumor, and chemotherapy is indicated for the treatment of stage IV MCC [6]. However, the effectiveness of chemotherapy in terms of survival benefit is difficult to assess from the literature [7]. Although chemotherapy may result in initial tumor regression, retrospective studies have shown overall survival to be less than 10 months and patients experience significant toxic effects [8-14]. No current consensus exists on the most effective treatment strategy, particularly in advanced tumor stages; however, there is now evidence indicating immunotherapy to be a promising approach in the treatment of MCC [15]. In that respect, PD-L1 has been shown to play a key role in the reactivation of the immune response against multiple cancers [16] and may provide a feasible target for intervention.

Avelumab is a fully human IgG1 monoclonal antibody targeting and blocking PD-L1 interaction with its receptor PD-1 [15]. In the primary analysis of a single-arm, Phase II study, JAVELIN Merkel 200 (Part A, 2L+ metastatic MCC [mMCC] patients), avelumab therapy resulted in an objective response rate of 33\% [17]. The proportion of

Future $\because \%$ Medicine 
patients who were progression-free at 6 months was 40\%, which is higher than observed with chemotherapy as reported in a retrospective review of stage IV MCC patients [9]. Avelumab has recently been approved by the US FDA and the European Commission for the treatment of mMCC and is the first drug with an approved indication in this tumor type [18].

To date, there is little knowledge about quality of life (QoL) and the impact of disease progression in patients with mMCC. A recent qualitative analysis of patient interviews, conducted alongside the avelumab Phase II trial, showed that for most patients, MCC was symptomless and painless prior to, as well as following diagnosis [19]. Although patients were for the most part able to conduct activities of everyday life, they reported a significant psychological impact. Patients reported a clear benefit with avelumab treatment, as reflected by improved tumor status as well as improved physical and mental health. Patients reported high satisfaction with avelumab, which they described as less debilitating and/or better tolerated than chemotherapy or radiotherapy.

The importance of patient-reported outcomes (PROs) as measured using existing validated instruments has been acknowledged by regulatory bodies [20,21]. Generic instruments can facilitate comparisons across populations, yet may lack sensitivity to detect changes in specific conditions [22,23]. Conversely, disease-specific instruments have an increased sensitivity to detect changes within a certain condition, but lack the potential to compare results across populations [24,25]. Thus, it is recommended to use a combination of both generic and disease-specific instruments [26].

Among generic instruments, the EuroQol - 5 Dimensions (EQ-5D) questionnaire has been widely used in oncology [27] and has been shown to be reliable and responsive and to have content as well as construct validity. No disease-specific instruments exist to specifically capture health-related quality of life (HRQoL) in patients with MCC. However, the Functional Assessment of Cancer Therapy - Melanoma (FACT-M) has shown promising characteristics for patients with malignant melanomas and was reported to have significant correlations with other instruments measuring similar constructs, such as the European Organization for Research and Treatment of Cancer - Quality of Life Questionnaire (EORTC-QLQ) Melanoma Module and the Profile of Mood States. Furthermore, the FACT-M has been shown to distinguish between disease stages, showing significant decreases in scores of patients with advanced disease compared with patients in early stages of the disease [28]. Using data from the JAVELIN Merkel 200 Phase II trial, a psychometric validation of the FACT-M in MCC has been carried out, demonstrating acceptable psychometric properties in this patient population [29].

The purpose of this study was to assess the association between HRQoL and disease progression in patients with mMCC treated with avelumab.

\section{Materials \& methods \\ Study design}

This study used data collected in Part A of trial EMR 100070-003/NCT02155647/ JAVELIN Merkel 200, a single-arm, open-label, multicenter, international Phase II study in patients with distant mMCC. Patients enrolled in the trial were required to have histologically confirmed stage IV MCC, must have received at least one line of chemotherapy for the treatment of mMCC and must have progressed after the most recent line of chemotherapy. Eligible patients were adults aged at least 18 years who had an Eastern Cooperative Oncology Group performance score of 0 or 1 at trial entry, an estimated life expectancy of more than 12 weeks, at least one unidimensional measurable lesion by Response Evaluation Criteria In Solid Tumors (RECIST) version 1.1 and adequate hematological, hepatic and renal function. Details on the definitions of these inclusion criteria as well as additional patient eligibility criteria are reported elsewhere [15].

Patients received avelumab at a dose of $10 \mathrm{mg} / \mathrm{kg}$ as a 1-h intravenous infusion every 2 weeks until significant clinical deterioration, unacceptable toxicity or any protocol-specified criterion for withdrawal from the trial or trial drug was fulfilled.

Data reported in this study are based on the protocol specified analysis with a cut-off date on 3 September 2016, 12 months after start of study treatment of the last patient.

\section{Patient reported outcome study assessments}

PRO data were collected electronically at sites throughout the treatment period (at baseline, week 7 and then every 6 weeks) and at the end-of-treatment visit.

HRQoL was assessed with the melanoma-specific FACT-M questionnaire and the generic 5-level EQ-5D (EQ5D-5L) questionnaire. 


\section{FACT-M}

The FACT-M comprises 51 items grouped into nine multi-item scores, including six subscale scores and three summary scores [24,30]. The six subscales consist of four subscales from the FACT - General (FACT-G; physical wellbeing, social wellbeing, emotional wellbeing, functional wellbeing), one melanoma scale and one melanoma surgery scale. The three summary scores include the FACT-M trial outcome index (TOI), the FACT-G total score and the FACT-M total score.

\section{$E Q-5 D$}

The EQ-5D is a self-administered, generic, preference-based HRQoL instrument developed by the EuroQol group [31,32]. The five level version (EQ-5D-5L) that was used in this study includes five single-item dimensions with five response levels each and a vertical visual analog scale (VAS) for the patients to rate their current health state. The VAS ranges from 0 (worst imaginable health state) to 100 (best imaginable health state). Health states defined by responses to the five items can be transformed into a single utility value (EQ-5D index) using a scoring algorithm that is based on valuations of representative general population samples. In this study, utility values were calculated using the country-specific value set for the USA, which is based on the cross-walk methodology developed by the EuroQol group [33].

\section{Tumor response}

Tumor response was determined by radiological assessment by an independent review committee per RECIST version 1.1 [34]. Response status was categorized as nonprogressed health states (stable disease [SD], partial response or complete response) versus progressive disease (PD). For each HRQoL assessment, tumor response status was determined as the result of the tumor response evaluation (based on radiological evaluation and physical examination of skin lesions, if appropriate) that took place within a time window of \pm 7 days around the date of the HRQoL assessment. HRQoL data that were collected outside of these time windows around tumor response evaluations were excluded from the analysis, as were observations when a patient's tumor response was 'not evaluable'.

\section{Statistical analyses}

Several different approaches were applied to analyze the association between clinical response and patients' health status.

\section{Tumor size \& change from baseline in HRQoL \& utility scores}

First, the association between percentage reduction of tumor size and changes from baseline in EQ-5D and FACT$\mathrm{M}$ scores was evaluated by calculating correlation coefficients and simple linear regressions at week 7 . This time point was chosen for sample size reasons, and because it enabled the most balanced mix of patients with versus without disease progression, providing sufficient variation in tumor size reduction across patients.

\section{Tumor response \& change from baseline in HRQoL \& utility scores}

Second, the association between tumor response and change in FACT-M and EQ-5D scores from baseline was estimated by using linear mixed models (LMMs). LMMs take into account that patients have repeated measurements over time by introducing a random effect for each patient. One LMM was fitted for change from baseline in each of the six FACT-M subscales and each of the three FACT-M summary scores, as well as for change from baseline in EQ-VAS and EQ-5D utility scores. These analyses were based on data from visits between baseline and week 49. Each LMM included the following covariates: baseline value of the considered score, number of visit and a time-dependent variable on response status (nonprogressed vs progressed). Analyses included patients who had an evaluable score at baseline and at least one postbaseline assessment.

\section{Tumor response \& mean utility scores}

Third, further LMMs were fitted to estimate adjusted mean EQ-5D utilities (rather than mean change from baseline) by treatment response at the corresponding visit (nonprogressed vs progressed). Since utilities based on general population preferences from the UK are most frequently used in literature and are typically also used by most countries without their own value set, these analyses were also conducted for the UK value set developed by Devlin et al. [35]. These LMMs were fitted to EQ-5D utility scores from all available time points, including baseline. The model included response status as the only fixed effects covariate. From this regression model, adjusted mean 
utilities were derived for nonprogressed and progressed observations, respectively. Response status at baseline was set to SD.

\section{Adjustments for the impact of adverse events on mean utility scores}

To assess the impact of adverse events (AEs) on EQ-5D utilities and to provide estimates of mean EQ-5D utilities adjusted for the effect of AEs, a further binary, time-dependent covariate was added to the LMM, reflecting whether a patient had at least one AE ongoing at the time of EQ-5D assessment. Presence of AEs was determined based on reported start and end dates of each individual AE. From this additional regression model, mean utilities for nonprogressed versus progressed health states adjusted for AEs were obtained by predicting utilities for an average patient in this health state assumed to have no AEs. In addition, the average loss in EQ-5D utilities associated with experiencing an $\mathrm{AE}$ ('disutility') was obtained as the regression coefficient of the AE variable in the LMM. A number of different types of AEs were considered including AEs of grade 3 or 4, treatment-related AEs of any grade or serious AEs.

Statistical analyses were performed using SAS software for Windows version 9.4 (SAS Institute, Inc., NC, USA).

\section{Results}

\section{Patient population}

Among the 88 patients who received at least one dose of trial treatment, $72(81.8 \%)$ patients completed at least one item of the EQ-5D and 70 patients completed at least one item of the FACT-M questionnaires, respectively, at baseline. The number of patients who completed the EQ-5D (FACT-M) questionnaire was 54 (53) at week 7, 43 (42) at week 13 and 32 (31) at week 25.

Median time since diagnosis of metastatic disease was 10.4 months (range: 1.5-159.0) - see Table 1. The primary tumor site was skin in most patients. Patients had a median follow-up time of 16.4 months (range: 12.1-25.4) from first trial treatment to data analysis cut-off date. The proportion of patients who were progression-free at 6 months was $40.0 \%$ (95\% CI: $29-50)$.

Mean FACT-G total score at baseline was 78.2 (SD: 19.28); mean FACT-M total score was 128.7 (SD: 25.24). EQ-5D VAS at baseline was 65.2. Mean EQ-5D utility in the trial at baseline was 0.799 (SD: 0.155) for the US tariff and 0.823 (SD: 0.196) for the UK tariff, respectively.

\section{Correlation between reduction in tumor size $\&$ health status}

A positive association between percentage reduction in tumor size and patients' HRQoL (change from baseline) was generally observed at week 7 . The largest correlation coefficients were observed among the functional wellbeing (correlation coefficient $[\rho]=-0.468$ [95\% CI: $-0.679-0.173]$ ), FACT-M TOI $(\rho=-0.364$ [95\% CI: $-0.607-$ 0.050]), FACT-M total $(\rho=-0.364$ [95\% CI: $-0.607-0.050])$, FACT-G total $(\rho=-0.338$ [95\% CI: $-0.588-$ $0.021])$ and EQ-5D index score $(\rho=-0.318$ [95\% CI: $-0.570-0.004])$. Derived from this association, based on a univariate linear regression model, a $30 \%$ decrease in tumor size (which would qualify for partial response for target lesions according to RECIST) corresponds to a predicted mean 5.5-point improvement on the FACT-M total score (extrapolated from the regression coefficient of 0.184 [95\% CI: $-0.340-0.027$ ] per percentage point). This is considered a clinically meaningful improvement on this QoL score, assuming that the minimally important difference (MID) of the FACT-M obtained from melanoma patients is also valid for the MCC population [29,36]. Correlation coefficients calculated between tumor size change and change in EQ-5D VAS score were small ( $\rho$ $=-0.188)$, indicating no clear association.

\section{Association between tumor response \& change in HRQoL scores from baseline}

Mean change from baseline in FACT-M and EQ-5D scores by tumor response status (nonprogressed vs progressed) are shown in Table 2. Mean differences in change from baseline scores between non-PD and PD were in the range of published MIDs for melanoma subscale (difference $=2.79, \mathrm{p}=0.005$ ), melanoma surgery scale (difference $=$ $1.48, \mathrm{p}=0.089)$, TOI (difference $=5.68, \mathrm{p}=0.004)$, FACT-G total score $($ difference $=4.37, \mathrm{p}=0.022)$, FACT-M total score (difference $=7.03, \mathrm{p}=0.006$ ), EQ-5D VAS (difference $=7.04, \mathrm{p}=0.024$ ) and EQ-5D utility score (difference $=0.07, \mathrm{p}=0.003$ ). 


\begin{tabular}{|c|c|}
\hline Baseline characteristics & Patients $(n=88)$ \\
\hline Age (years), median (range): & $72.5(33-88)$ \\
\hline$-<65, \mathrm{n}(\%)$ & $22(25)$ \\
\hline$-\geq 65, \mathrm{n}(\%)$ & $66(75)$ \\
\hline \multicolumn{2}{|l|}{ Sex, n (\%): } \\
\hline - Male & $65(74)$ \\
\hline - Female & $23(26)$ \\
\hline \multicolumn{2}{|l|}{ Site of primary tumor, $\mathrm{n}(\%)$ : } \\
\hline - Skin & $67(76)$ \\
\hline - Lymph node & $12(14)$ \\
\hline - Other & $2(2)$ \\
\hline - Missing & $7(8)$ \\
\hline \multicolumn{2}{|l|}{ Visceral disease at study entry, $\mathrm{n}(\%)$ : } \\
\hline - Present & $47(53)$ \\
\hline - Absent & $41(47)$ \\
\hline \multicolumn{2}{|l|}{ ECOG performance status score, $n(\%)$ : } \\
\hline-0 & $49(56)$ \\
\hline-1 & $39(44)$ \\
\hline Time since first diagnosis (months), median (range) & $19.8(2.9-159.0)$ \\
\hline Time since first diagnosis of metastatic disease (months), median (range) & $10.4(1.5-159.0)$ \\
\hline \multicolumn{2}{|l|}{ Number of previous systematic anticancer treatments, n (\%): } \\
\hline-1 & $52(59)$ \\
\hline-2 & $26(30)$ \\
\hline-3 & $7(8)$ \\
\hline$-\geq 4$ & $3(3)$ \\
\hline \multicolumn{2}{|l|}{ Follow-up/efficacy outcomes } \\
\hline Follow-up (months), median (range) & $16.4(12.1-25.4)$ \\
\hline Progression-free survival rate at 12 months & $30 \%(95 \% \mathrm{Cl}: 21-41)$ \\
\hline Overall survival rate at 12 months & $52 \%(95 \% \mathrm{Cl}: 41-62)$ \\
\hline \multicolumn{2}{|l|}{ Confirmed best overall response, $\mathrm{n}(\%)$ : } \\
\hline - Complete response (CR) & $10(11)$ \\
\hline - Partial response (PR) & $19(22)$ \\
\hline - Stable disease & $9(10)$ \\
\hline - Progressive disease & $32(36)$ \\
\hline - Nonevaluable & $18(20)$ \\
\hline Objective response rate $(\mathrm{CR}+\mathrm{PR}), \%(95.9 \% \mathrm{Cl})^{\dagger}$ & $33.0 \%(95 \% \mathrm{Cl}: 23.3-43.8)$ \\
\hline $\begin{array}{l}\text { Data are median (range) or } \mathrm{n}(\%) \text { if not stated otherwise. } \\
{ }^{\dagger} \text { Exact confidence interval using the Clopper-method. } \\
\text { ECOG: Eastern Cooperative Oncology Group. }\end{array}$ & \\
\hline
\end{tabular}

\section{Mean EQ-5D utility by tumor response}

The LMMs that were fitted to estimate mean EQ-5D utility by tumor response (progressed vs nonprogressed) included a total of 322 observations from 70 patients. Of those, 267 observations were collected before documented disease progression and 55 observations were collected at or after disease progression. Mean utilities predicted from the LMM for progression and nonprogression health states are shown in Table 3. Estimates adjusted for the impact of AEs are also presented.

Utility based on US and UK value sets were 0.8024 and 0.8269 in the nonprogression health state, respectively. Utility values based on US and UK value sets were 0.7352 and 0.7415 in the progression health state, respectively. Differences between health states ( 0.067 for US value set $[p=0.0002]$; 0.085 for UK value set $[p<0.0001]$ ) were statistically significant and clinically relevant, based on published estimates of MID of 0.06 for the US tariff and 


\begin{tabular}{|c|c|c|c|c|c|c|c|c|}
\hline \multirow[t]{3}{*}{ FACT-M subscales and summary scores } & \multirow[t]{3}{*}{ n } & \multicolumn{4}{|c|}{ LS means (overall response status by IERC per RECIST) } & \multirow{3}{*}{$\begin{array}{l}\text { Difference in } \\
\text { estimate }\end{array}$} & \multirow[t]{3}{*}{ p-value } & \multirow{3}{*}{$\begin{array}{l}\text { Published MIDs in } \\
\text { melanoma } \\
\text { population }\end{array}$} \\
\hline & & \multicolumn{2}{|c|}{ Nonprogressed (CR/PR/SD) } & \multicolumn{2}{|c|}{ Progressed (PD) } & & & \\
\hline & & Estimate & p-value & Estimate & p-value & & & \\
\hline \multicolumn{9}{|l|}{ FACT-G subscales } \\
\hline PWB & 172 & -0.39 & 0.544 & -1.57 & $0.023^{*}$ & 1.18 & 0.080 & $2.00-3.00^{\dagger}$ \\
\hline SWB & 172 & -1.83 & $0.005^{*}$ & -1.89 & $0.009^{*}$ & 0.06 & 0.941 & - \\
\hline EWB & 172 & 1.70 & $0.001^{*}$ & 0.18 & 0.743 & 1.52 & $0.008^{*}$ & $2.00^{\dagger}$ \\
\hline FWB & 172 & 0.67 & 0.279 & -1.12 & 0.101 & 1.79 & $0.015^{*}$ & $2.00-3.00^{\dagger}$ \\
\hline \multicolumn{9}{|l|}{ Melanoma-specific subscales } \\
\hline MS & 172 & 1.49 & 0.081 & -1.30 & 0.162 & 2.79 & $0.005^{*}$ & $2.00-4.00^{\ddagger}$ \\
\hline MSS & 172 & -0.05 & 0.953 & -1.52 & 0.070 & 1.48 & 0.089 & $1.00-2.00^{\ddagger}$ \\
\hline \multicolumn{9}{|l|}{ Summary scales } \\
\hline FACT-M TOI & 172 & 1.66 & 0.360 & -4.01 & $0.039^{*}$ & 5.68 & $0.004^{*}$ & $5.00-9.00^{\ddagger}$ \\
\hline FACT-G total score & 172 & -0.07 & 0.970 & -4.44 & $0.020^{*}$ & 4.37 & $0.022^{*}$ & $3.00-7.00^{\dagger}$ \\
\hline FACT-M total score & 174 & 1.39 & 0.558 & -5.64 & $0.027^{*}$ & 7.03 & $0.006^{*}$ & - \\
\hline \multicolumn{9}{|l|}{ EQ-5D } \\
\hline VAS & 174 & 4.39 & $0.024^{\star}$ & -2.64 & $0.325^{*}$ & 7.04 & $0.024^{\star}$ & $7.00^{\S}$ \\
\hline Utility (US value set) & 172 & -0.01 & 0.780 & -0.08 & $0.002^{*}$ & 0.07 & $0.003^{*}$ & $0.06^{\S}$ \\
\hline \multicolumn{9}{|c|}{ 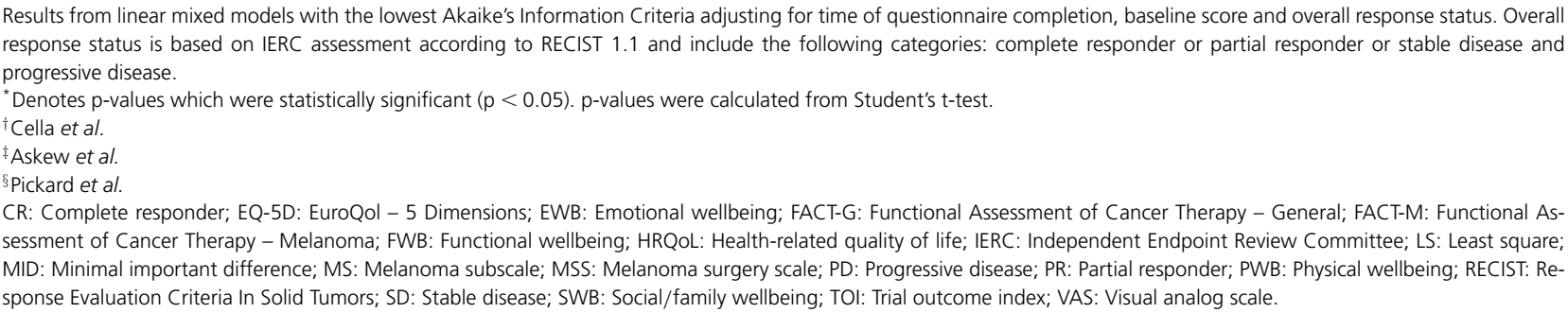 } \\
\hline
\end{tabular}

0.07 or 0.09 for the UK tariff $[37,38]$. The MID is the smallest change in outcome that a patient would identify as important.

\section{Impact of AEs on mean EQ-5D utility}

Patients with an ongoing AE of grade 3 or 4 at the time of the EQ-5D assessment had on average only slightly lower EQ-5D utility scores than patients without AEs (-0.033 [95\% CI: -0.075-0.008] for the US value set, and -0.033 [95\% CI: -0.080-0.015] for the UK value set, respectively) - see Table 4. These differences were neither clinically meaningful nor statistically significant $(\mathrm{p}=0.1113$ and $\mathrm{p}=0.1779$, respectively). Disutilities for treatment-related AEs of any grade were even smaller (-0.004 and -0.005 for US and UK value set, respectively). Only serious treatment-emergent AEs (13 observations) were associated with a clinically meaningful loss in utility (-0.061 for US value set; -0.098 for UK value set).

\section{Discussion}

Using data from the Phase II trial JAVELIN Merkel 200, these analyses explored the association between tumor response and HRQoL in patients with mMCC treated with avelumab after treatment with at least one line of chemotherapy. Results show that reduction in tumor size correlates with improvements in patients' health status, as measured by both generic and skin cancer specific HRQoL instruments. Results also demonstrated significantly higher HRQoL and utility for patients with non-PD compared with PD, measured as per RECIST criteria. Clinically meaningful differences, of similar magnitude to previously published MIDs, [36,39] were observed for most FACT-M changes from baseline scores.

Over the last decades, PROs have become an important end point in metastatic oncology and/or melanoma trials and are frequently used in evaluations by regulatory and reimbursement authorities [20,21]. PROs provide important patient perspectives on the disease and the treatment received that are not captured by traditional efficacy data or 
Table 3. Mean EuroQol - 5 Dimensions utility between disease progressed and nonprogressed patients.

\begin{tabular}{|c|c|c|c|c|c|}
\hline \multirow[t]{2}{*}{ EQ-5D utility } & \multirow[t]{2}{*}{ Statistics } & \multicolumn{2}{|c|}{ Response status (based on RECIST 1.1) } & \multirow[t]{2}{*}{ Difference $^{\dagger}$} & \multirow[t]{2}{*}{$\mathrm{p}$-value } \\
\hline & & Nonprogressed (CR/PR/SD) & Progressed (PD) & & \\
\hline \multicolumn{6}{|l|}{ US value set } \\
\hline \multirow[t]{2}{*}{ Observed values } & $\mathrm{n}^{\ddagger}$ & 267 & 55 & - & - \\
\hline & Min; $\max ^{\S}$ & $0.204 ; 1.000$ & $0.126 ; 1.000$ & - & - \\
\hline \multirow{2}{*}{$\begin{array}{l}\text { Adjusted means from LMM (main } \\
\text { analysis) }\end{array}$} & Mean $(95 \% \mathrm{Cl})$ & $0.8024(0.7668-0.8380)$ & $0.7352(0.6922-0.7781)$ & $0.0672(0.0317-0.1028)$ & 0.0002 \\
\hline & SE & 0.01787 & 0.02172 & 0.01808 & \\
\hline \multirow{2}{*}{$\begin{array}{l}\text { Adjusted means from LMM } \\
\text { (controlled for grade } 3 / 4 \text { AEs) }\end{array}$} & Mean $(95 \% \mathrm{Cl})$ & $0.8057(0.7704-0.8410)$ & $0.7490(0.7033-0.7946)$ & $0.0567(0.0189-0.0946)$ & 0.0034 \\
\hline & SE & 0.01769 & 0.02312 & 0.01922 & \\
\hline \multirow{2}{*}{$\begin{array}{l}\text { Adjusted means from LMM } \\
\text { (controlled for treatment-related } \\
\text { AEs) }\end{array}$} & Mean $(95 \% \mathrm{Cl})$ & $0.8034(0.7667-0.8401)$ & $0.7375(0.6901-0.7848)$ & $0.0659(0.0286-0.1033)$ & 0.0006 \\
\hline & SE & 0.01843 & 0.02398 & 0.01898 & \\
\hline \multicolumn{6}{|l|}{ UK value set } \\
\hline \multirow[t]{2}{*}{ Observed values } & $\mathrm{n}^{\ddagger}$ & 267 & 55 & - & - \\
\hline & Min; $\max ^{\S}$ & $-0.165 ; 1.000$ & $0.007 ; 1.000$ & - & - \\
\hline \multirow{2}{*}{$\begin{array}{l}\text { Adjusted means from LMM (main } \\
\text { analysis) }\end{array}$} & Mean $(95 \% \mathrm{Cl})$ & $0.8269(0.7842-0.8696)$ & $0.7415(0.6907-0.7923)$ & $0.0854(0.0445-0.1263)$ & $<0.0001$ \\
\hline & SE & 0.02140 & 0.02567 & 0.02079 & \\
\hline \multirow{2}{*}{$\begin{array}{l}\text { Adjusted means from LMM } \\
\text { (controlled for grade } 3 / 4 \text { AEs) }\end{array}$} & Mean $(95 \% \mathrm{Cl})$ & $0.8302(0.7879-0.8725)$ & $0.7550(0.7012-0.8088)$ & $0.0752(0.0316-0.1187)$ & 0.0008 \\
\hline & SE & 0.02117 & 0.02722 & 0.02212 & \\
\hline \multirow{2}{*}{$\begin{array}{l}\text { Adjusted means from LMM } \\
\text { (controlled for treatment-related } \\
\text { AEs) }\end{array}$} & Mean $(95 \% \mathrm{Cl})$ & $0.8283(0.7844-0.8722)$ & $0.7447(0.6890-0.8005)$ & $0.0836(0.0406-0.1266)$ & 0.0002 \\
\hline & SE & 0.02202 & 0.02823 & 0.02185 & \\
\hline \multicolumn{6}{|c|}{$\begin{array}{l}\text { p-values were calculated form Student's t-tests. } \\
\text { †MID = } 0.06 \text { for US tariff (Pickard et al.) and } 0.07-0.09 \text { for UK tariff (Pickard et al.; Walters et al.). } \\
\text { FUnit of analysis is EQ-5D measurement not patient, so } n>\text { number of patients. } \\
\text { sObserved data, not based on linear mixed model. Overall response status is based on IERC assessment according to RECIST } 1.1 \text { and includes the following categories: complete } \\
\text { responder or partial responder or stable disease (CR/PR/SD) and PD. } \\
\text { AE: Adverse event; CR: Complete responder; EQ-5D: EuroQol - } 5 \text { Dimensions; IERC: Independent Endpoint Review Committee; LMM: Linear mixed model; MID: Minimally } \\
\text { important difference; PD: Progressive disease; PR: Partial responder; RECIST: Response Evaluation Criteria In Solid Tumors; SD: Stable disease; SE: Standard error. }\end{array}$} \\
\hline
\end{tabular}

Table 4. Disutilities for different types of adverse events - adjusted for the effect of disease progression.

\begin{tabular}{|c|c|c|c|c|c|c|c|c|c|}
\hline \multicolumn{2}{|c|}{ Type of adverse event } & \multicolumn{4}{|c|}{ US value set } & \multicolumn{4}{|c|}{ UK value set } \\
\hline Name & n (with AE) ${ }^{\dagger}$ & $\begin{array}{l}\text { Mean } \\
\text { disutility }\end{array}$ & SE & $95 \% \mathrm{Cl}$ & p-value & $\begin{array}{l}\text { Mean } \\
\text { disutility }\end{array}$ & SE & $95 \% \mathrm{Cl}$ & p-value \\
\hline $\begin{array}{l}\text { Grade } 3 \text { or } 4 \\
\mathrm{AE}\end{array}$ & 40 & -0.03341 & 0.02092 & $\begin{array}{l}-0.07458- \\
0.007762\end{array}$ & 0.1113 & -0.03250 & 0.02407 & $\begin{array}{l}-0.07988- \\
0.01487\end{array}$ & 0.1779 \\
\hline $\begin{array}{l}\text { Treatment- } \\
\text { related } A E \text { of } \\
\text { any grade }\end{array}$ & 154 & -0.00360 & 0.01559 & $\begin{array}{l}-0.03427- \\
0.02708\end{array}$ & 0.8177 & -0.00502 & 0.01794 & $\begin{array}{l}-0.04032- \\
0.03029\end{array}$ & 0.7799 \\
\hline $\begin{array}{l}\text { Treatment- } \\
\text { emergent } A E \\
\text { of any grade }\end{array}$ & 237 & -0.02292 & 0.01494 & $\begin{array}{l}-0.05232- \\
0.006489\end{array}$ & 0.1262 & -0.02823 & 0.01713 & $\begin{array}{l}-0.06196- \\
0.00549\end{array}$ & 0.1005 \\
\hline $\begin{array}{l}\text { Serious } \\
\text { treatment- } \\
\text { emergent } \\
\text { AE }\end{array}$ & 13 & -0.06073 & 0.03664 & $\begin{array}{l}-0.1328- \\
0.01137\end{array}$ & 0.0985 & -0.09847 & 0.04207 & $\begin{array}{l}-0.1812- \\
0.01570\end{array}$ & 0.0199 \\
\hline $\begin{array}{l}\text { Treatment- } \\
\text { emergent } \\
\text { grade } 3 \text { or } 4 \\
\text { AE }\end{array}$ & 37 & -0.02405 & 0.02127 & $\begin{array}{l}-0.06591- \\
0.01781\end{array}$ & 0.2591 & -0.01668 & 0.02444 & $\begin{array}{l}-0.06479- \\
0.03143\end{array}$ & 0.4955 \\
\hline \multicolumn{10}{|c|}{$\begin{array}{l}\text { Disutilities were estimated from a linear mixed model including response status (progressed vs nonprogressed) and the AE variable as covariates. } \\
\text { †Unit of analysis is HRQoL measurement not the patient, so } n>\text { number of patients. } \\
\text { ¥Negative number indicates a disutility. } \\
\text { AE: Adverse events; HRQoL: Health-related quality of life; SE: Standard error. }\end{array}$} \\
\hline
\end{tabular}


AE reporting. Currently, no validated tools exist to capture HRQoL in patients with MCC. The FACT-M had been developed to address disease-specific QoL concerns among patients with melanoma, and it has subsequently been validated for use in patients with all stages of the disease [24,30]. MCC and melanoma are both aggressive skin malignancies; despite some differences between MCC and melanoma, including worse prognosis for MCC, the content of the FACT-M seems appropriate to assess QoL in patients with MCC. This was further confirmed by a thorough psychometric validation of the FACT-M in the JAVELIN Merkel 200 trial population [29].

Avelumab has been shown to achieve meaningful clinical benefit in mMCC with an ORR of 33.0\% (95\% CI: 23.3-43.8), and Kaplan-Meier estimated proportion of responses with at least 6 months' duration was 93\% (95\% CI: 74-98). The 6-month progression-free survival rate was 40\% (95\% CI: 29-50). The analyses reported here add further evidence to support the value of avelumab from the patient perspective, indicating that delaying disease progression translates into prolonging maintenance of HRQoL. Whereas PD was associated with significant and clinically meaningful reductions in HRQoL and utility, patients in nonprogressed health states (including SD and partial or complete response) had stable HRQoL scores or experienced small - although not clinically meaningful - improvements in HRQoL. In contrast, studies have shown that treatment with chemotherapy often comes at the cost of decrements in HRQoL caused by side effects of therapy [40,41].

The analyses of EQ-5D scores adjusted for the impact of AEs showed that the majority of side effects of avelumab do not have a detrimental impact on HRQoL. This is in line with the fact that most AEs related to avelumab were low grade, consisting mostly of fatigue and infusion-related reaction. Taken together, this indicates that avelumab is well tolerated and that its safety profile was manageable, whereas previous studies in patients with mMCC treated with chemotherapy have shown high rates of significant toxicities [8-11].

Baseline HRQoL scores in this trial of mMCC patients were lower than average HRQoL scores reported for an adult US general population, reflecting the burden that mMCC has on patient health status [42], but they were similar to reference values reported for an adult metastatic cancer population [37,42,43]. Mean FACT-M scores were lower than reported in a previous study of patients with stage IV melanoma [24,44]. This could be due to the fact that MCC is in general a more aggressive tumor than melanoma [6]. However, differences between the two studies at baseline could also be impacted by other characteristics of the underlying populations; such as type of treatment or patient age. Mean age of melanoma patients by disease stage was not reported, however, it is likely that patients with stage IV melanoma were younger than those with mMCC, as median age in the overall melanoma study population (including stage I-IV) was only 52 years, as compared with 72.5 in the JAVELIN Merkel 200 trial [24].

The observed decline in utility associated with disease progression in this study is consistent with (and even more pronounced than) the results from similar studies in other indications. For example, Griebsch et al. examined whether disease progression is associated with HRQoL using empirical data from two randomized controlled trials in non-small-cell lung cancer [45]. It was found that disease progression was associated with a decline in utility of 0.06 points (derived with the EQ-5D using the UK general population value set). Singh and colleagues examined the association between disease progression and EQ-5D utilities obtained by mapping from the FACT-G questionnaire in patients with neuroendocrine tumors of gastrointestinal or lung origin [46]; they reported preprogression utilities of 0.826 (for a US value set) and 0.779 (for a UK value set), respectively, versus postprogression utilities of 0.795 (US value set) and 0.725 (UK value set), respectively. Although statistically significant, this difference was not clinically relevant based on published MIDs of 0.06 for the US tariff and 0.07-0.09 for the UK tariff, respectively [37,38].

The differences between mean utilities estimated by the UK and US tariff are consistent with previous studies that have shown that EQ-5D index scores are slightly higher when using US weights as compared with UK weights [47].

The association between disease progression and decline in HRQoL demonstrated in this analysis, has important healthcare policy and clinical implications. In the era of patient-centered medicine, end points such as HRQoL are increasingly important and should be part of the larger context of clinical benefit. Our results show that disease progression is associated with HRQoL and utility, supporting the use of progression-free survival as a patient relevant outcome in clinical trials in patients with MCC. The HRQoL and utility values reported in this study may be used in health technology appraisals. In particular, empirical estimates of mean utility by tumor response are an important input parameter in health economic models that are built to simulate the effectiveness of treatments over a lifetime horizon [48]. Health economic models are required by many national regulatory bodies as part of their Health Technology Assessment.

In the JAVELIN Merkel 200 trial, collection of HRQoL data was only required until disease progression. This resulted in scarcity of data post disease progression; however, there was at least one assessment at or after disease progression for each patient with PD. The collected data appears adequate for the derivation of robust estimates 
for HRQoL and utility postprogression. If HRQoL further worsens after disease progression, then the difference between pre- and postprogression may even have been underestimated in this study. Another limitation is that patients may have rescheduled their visits if they were bothered by severe AEs, which may lead to an underestimation of disutilities related to AEs.

In conclusion, this study showed that patients on avelumab who are progression-free have significantly better HRQoL and health status than patients after disease progression. This indicates that durable response rates observed with avelumab would also be expected to translate into prolonging maintenance of HRQoL. The impact of AEs on HRQoL and utility during treatment with avelumab was minimal, suggesting an acceptable safety profile from a patient perspective.

\section{Summary points}

- This study is one of the first to describe health-related quality of life (HRQoL) in patients with metastatic Merkel cell carcinoma (MCC), a rare and aggressive cutaneous malignancy.

- In the JAVELIN Merkel 200 trial, 88 patients with metastatic MCC were treated with avelumab, a fully human IgG1 monoclonal antibody targeting and blocking PD-L1 interaction with its receptor PD-1.

- Reduction of tumor size experienced under treatment with avelumab correlates with improvements in patients' health status, as measured by both generic and skin cancer specific HRQoL instruments.

- A $30 \%$ decrease in tumor size (which would qualify for partial response for target lesions according to the Response Evaluation Criteria In Solid Tumors [RECIST]) corresponds to a clinically meaningful improvement of 5.5 points on the Functional Assessment of Cancer Therapy - Melanoma (FACT-M) total score.

- Progressive disease (as per RECIST) is associated with statistically significant and clinically meaningful reductions in HRQoL and utility, while patients in nonprogressed health states had stable HRQoL or experienced small improvements in HRQoL.

- The majority of adverse events experienced under treatment with avelumab (including grade 3 or 4 adverse events) do not have a detrimental impact on HRQoL.

- Study results indicate that delaying disease progression under treatment with avelumab translates into prolonging maintenance of HRQoL.

- The association between disease progression (as per RECIST) and decline in HRQoL supports the use of progression-free survival as a patient-relevant outcome in clinical trials in MCC patients.

\section{Authors contributions}

$\mathrm{H}$ Kaufman participated in the study design and data interpretation and reviewed the manuscript. M Hunger conducted the statistical analyses. M Hennessy participated in the study design and data interpretation and reviewed the manuscript. M Schlichting participated in the study design and data interpretation and reviewed the manuscript. M Bharmal participated in the study design and data interpretation and reviewed the manuscript.

\section{Acknowledgements}

The authors wish to acknowledge sites and patients who participated in the clinical trial. C Storck, Mapi, provided support for scientific writing.

Financial \& competing interests disclosure

This study was funded by Merck KGaA. H Kaufman has received research grants from Amgen, Merck. H Kaufman has provided consulting for Amgen, Celldex, EMD Serono, Merck, Prometheus, Sanofi, Turnstone Biologics and participated in speaker's bureaus for Merck. H Kaufman has received honoraria from Amgen, Celldex, EMD Serono, Merck, Prometheus, Sanofi, Turnstone Biologics. M Hunger, Mapi employee, was paid consultant for Merck KGaA. M Hennessy is an employee of EMD Serono. M Schlichting and M Bharmal are employees of Merck KGaA, Darmstadt, Germany. The authors have no other relevant affiliations or financial involvement with any organization or entity with a financial interest in or financial conflict with the subject matter or materials discussed in the manuscript apart from those disclosed.

No writing assistance was utilized in the production of this manuscript. 
Ethical conduct of research

This study was performed in compliance with the ethical principles arising from the Declaration of Helsinki and all current local regulations. The study protocol was approved by Independent Ethics Committee or Institutional Review Board prior to the study launch at each site. Written informed consent was provided by patients or their legal representatives.

Open access

This work is licensed under the Attribution-NonCommercial-NoDerivatives 4.0 Unported License. To view a copy of this license, visit http://creativecommons.org/licenses/by-nc-nd/4.0/

\section{References}

Papers of special note have been highlighted as: $\bullet$ of interest; $\bullet \bullet$ of considerable interest

1 Heath M, Jaimes N, Lemos B et al. Clinical characteristics of Merkel cell carcinoma at diagnosis in 195 patients: the AEIOU features. J. Am. Acad. Dermatol. 58(3), 375-381 (2008).

2 List of rare cancers. www.rarecarenet.eu/rarecarenet/index.php/cancerlist

3 Fitzgerald TL, Dennis S, Kachare SD, Vohra NA, Wong JH, Zervos EE. Dramatic increase in the incidence and mortality from Merkel cell carcinoma in the United States. Am. Surg. 81(8), 802-806 (2015).

4 Becker JC. Merkel cell carcinoma. Ann. Oncol. 21(Suppl. 7), vii81-vii85 (2010).

5 Lemos BD, Storer BE, Iyer JG et al. Pathologic nodal evaluation improves prognostic accuracy in Merkel cell carcinoma: analysis of 5823 cases as the basis of the first consensus staging system. J. Am. Acad. Dermatol. 63(5), 751-761 (2010).

6 Schadendorf D, Lebbe C, Zur Hausen A et al. Merkel cell carcinoma: epidemiology, prognosis, therapy and unmet medical needs. Eur. J. Cancer 71, 53-69 (2017).

•• A very comprehensive review of epidemiology, clinical burden and current therapy for Merkel cell carcinoma (MCC).

7 National Comprehensive Cancer Network (NCCN). NCCN clinical practice guidelines in oncology: Merkel cell carcinoma. www.nccn.org/professionals/physician_gls/pdf/mcc.pdf

8 Sharma D, Flora G, Grunberg SM. Chemotherapy of metastatic Merkel cell carcinoma: case report and review of the literature. Am. J. Clin. Oncol. 14(2), 166-169 (1991).

9 Iyer JG, Blom A, Doumani R et al. Response rates and durability of chemotherapy among 62 patients with metastatic Merkel cell carcinoma. Cancer Med. 5(9), 2294-2301 (2016).

- An observational study reporting on clinical outcomes of patients with MCC treated with chemotherapy in a real-world setting. It can be used as a historical cohort for the JAVELIN Merkel 200 trial.

10 Voog E, Biron P, Martin JP, Blay JY. Chemotherapy for patients with locally advanced or metastatic Merkel cell carcinoma. Cancer 85(12), 2589-2595 (1999).

11 Tai PT, Yu E, Winquist E et al. Chemotherapy in neuroendocrine/Merkel cell carcinoma of the skin: case series and review of 204 cases. J. Clin. Oncol. 18(12), 2493-2499 (2000).

12 Satpute S, Ammakkanavar N, Einhorn L. Role of platinum-based chemotherapy for Merkel cell tumor in adjuvant and metastatic settings. J. Clin. Oncol. 32, 9049 (2014).

13 Becker J, Lorenz E, Haas G, Helwig C, Oksen D, Mahnke L. Evaluation of real world treatment outcomes in patients with metastatic Merkel cell carcinoma (MCC) following second line chemotherapy. Ann. Oncol. 26, 2602 (2016).

- A retrospective observational study assessing treatment outcomes among second line or later chemotherapy in a real-world setting to better understand treatment pathways and prognosis in distant metastatic MCC patients.

14 Cowey C, Mahnke L, Espirito J, Fox P, Helwig C, Oksen D. Real-world outcomes of patients with metastatic Merkel cell carcinoma treated with second-line or later chemotherapy in a community oncology setting in the United States. Value Health 19(7), A717 (2016).

15 Kaufman HL, Russell J, Hamid O et al. Avelumab in patients with chemotherapy-refractory metastatic Merkel cell carcinoma: a multicentre, single-group, open-label, Phase II trial. Lancet Oncol. 17(10), 1374-1385 (2016).

•• The main publication reporting clinical efficacy outcomes in the JAVELIN Merkel 200 trial, showing that avelumab was associated with durable responses and well tolerated.

16 Iwai Y, Ishida M, Tanaka Y, Okazaki T, Honjo T, Minato N. Involvement of PD-L1 on tumor cells in the escape from host immune system and tumor immunotherapy by PD-L1 blockade. Proc. Natl Acad. Sci. USA 99(19), 12293-12297 (2002).

17 Kaufman H, Russell J, Hamid O et al. Durable responses to avelumab (anti-PD-L1) in patients with Merkel cell carcinoma progressed after chemotherapy: 1-year efficacy update. Presented at: 2017 AACR Annual meeting; April 2-5 (Abstract CT079). Washington DC, USA 2017.

18 US FDA. Bavencio (avelumab). www.accessdata.fda.gov/scripts/cder/daf/index.cfm?event=BasicSearch.process 
19 Kaufmann H, Kraemer M, Dias Barbosa C, Lamber J, Mahnke L, Bharmal M. Patient perspectives on Merkel cell carcinoma (MCC) and its treatment with a novel agent (avelumab): findings from in-depth qualitative patient interviews. Presented at: ISPOR 19th Annual European Congress. Vienna, Austria, 29 October-2 November 2016.

20 EMA. Appendix 2 to the Guideline on the Evaluation of Anticancer Medicinal Products in Man. The Use of Patient-Reported Outcome (PRO)Measures in Oncology Studies. www.ema.europa.eu/docs/en_GB/document_library/Other/2016/04/WC500205159.pdf

21 US FDA. Guidance for Industry - Patient Reported Outcome Measures: Use in Medical Product Claims to Support Labeling Claims. www.fda.gov/downloads/drugs/guidances/ucm193282.pdf

22 Cella DF, Tulsky DS. Quality of life in cancer: definition, purpose, and method of measurement. Cancer Invest. 11(3), 327-336 (1993).

23 Langenhoff B, Krabbe P, Wobbes T, Ruers T. Quality of life as an outcome measure in surgical oncology. Br. J. Surg. 88, 643-652 (2001).

24 Cormier JN, Ross MI, Gershenwald JE et al.. Prospective assessment of the reliability, validity, and sensitivity to change of the Functional Assessment of Cancer Therapy-Melanoma questionnaire. Cancer 112(10), 2249-2257 (2008).

- A comprehensive study on the psychometric validation of the Functional Assessment of Cancer Therapy - Melanoma (FACT-M) questionnaire, showing that it is a reliable and valid instrument for patients with skin cancer that can be used for the assessment of health-related quality of life in clinical trials.

25 Lipscomb, Gotay C, Snyder C. Outcomes Assessment in Cancer: Measures, Methods, and Applications. Cambridge University Press, Cambridge, UK (2005).

26 Both H, Essink-Bot ML, Busschbach J, Nijsten T. Critical review of generic and dermatology-specific health-related quality of life instruments. J. Invest. Dermatol. 127(12), 2726-2739 (2007).

27 Schwenkglenks M, Matter-Walstra K. Is the EQ-5D suitable for use in oncology? An overview of the literature and recent developments. Expert Rev. Pharmacoecon. Outcomes Res. 16(2), 207-219 (2016).

28 Cormier JN, Cromwell KD, Ross MI. Health-related quality of life in patients with melanoma: overview of instruments and outcomes. Dermatol. Clin. 30(2), 245-254, viii (2012).

29 Bharmal M, Fofana F, Dias Barbosa C, Mahnke L, Schlichting M. Psychometric validation of the FACT-M questionnaire in patients with Merkel cell carcinoma. Presented at: ISPOR 22nd Annual International Meeting. Abstract PCN189, MA, USA, 20-24 May 2017.

-. A thorough psychometric validation study of the FACT-M in patients with metastatic MCC.

30 Cormier JN, Davidson L, Xing Y, Webster K, Cella D. Measuring quality of life in patients with melanoma: development of the FACT-melanoma subscale. J. Support. Oncol. 3(2), 139-145 (2005).

31 EuroQol - a new facility for the measurement of health-related quality of life. Health Policy 16(3), 199-208 (1990).

32 Herdman M, Gudex C, Lloyd A et al. Development and preliminary testing of the new five-level version of EQ-5D (EQ-5D-5L). Qual. Life Res. 20(10), 1727-1736 (2011).

33 Van Hout B, Janssen MF, Feng YS et al. Interim scoring for the EQ-5D-5L: mapping the EQ-5D-5L to EQ-5D-3L value sets. Value Health 15(5), 708-715 (2012).

- Describing how to calculate utility based on the 5-level EuroQol - 5 Dimensions questionnaire.

34 Eisenhauer EA, Therasse P, Bogaerts J et al. New response evaluation criteria in solid tumours: revised RECIST guideline (version 1.1). Eur. J. Cancer 45(2), 228-247 (2009).

35 Devlin N, Shah K, Feng Y, Mulhern B, Van Hout B. Valuing health-related quality of life: an EQ-5D-5L value set for England. Health Econ. doi:10.1002/hec.3564 (2017) (Epub ahead of print).

36 Askew RL, Xing Y, Palmer JL, Cella D, Moye LA, Cormier JN. Evaluating minimal important differences for the FACT-Melanoma quality of life questionnaire. Value Health 12(8), 1144-1150 (2009).

37 Pickard AS, Neary MP, Cella D. Estimation of minimally important differences in EQ-5D utility and VAS scores in cancer. Health Qual. Life Outcomes 5, 70 (2007).

38 Walters SJ, Brazier JE. Comparison of the minimally important difference for two health state utility measures: EQ-5D and SF-6D. Qual. Life Res. 14(6), 1523-1532 (2005).

39 Cella D, Hahn EA, Dineen K. Meaningful change in cancer-specific quality of life scores: differences between improvement and worsening. Qual. Life Res. 11(3), 207-221 (2002).

40 Cornish D, Holterhues C, Van De Poll-Franse LV, Coebergh JW, Nijsten T. A systematic review of health-related quality of life in cutaneous melanoma. Ann. Oncol. 20(Suppl. 6), vi51-vi58 (2009).

41 Schadendorf D, Dummer R, Hauschild A et al. Health-related quality of life in the randomised KEYNOTE-002 study of pembrolizumab versus chemotherapy in patients with ipilimumab-refractory melanoma. Eur. J. Cancer 67, 46-54 (2016).

42 Pearman T, Yanez B, Peipert J, Wortman K, Beaumont J, Cella D. Ambulatory cancer and US general population reference values and cutoff scores for the functional assessment of cancer therapy. Cancer 120(18), 2902-2909 (2014).

43 Janssen B, Szende A. Population norms for the EQ-5D. In: Self-Reported Population Health: An International Perspective based on EQ-5D. Szende A, Janssen M, Cabases J (Eds). Springer, Dordrecht, The Netherlands, 19-30 (2014). 
44 Askew RL, Swartz RJ, Xing Y et al. Mapping FACT-melanoma quality-of-life scores to EQ-5D health utility weights. Value Health 14(6), 900-906 (2011).

45 Griebsch I, Palmer M, Fayers PM, Ellis S. Is progression-free survival associated with a better health-related quality of life in patients with lung cancer? Evidence from two randomised trials with afatinib. BMJ Open 4(10), e005762 (2014).

46 Pavel ME, Singh S, Strosberg JR et al. Health-related quality of life for everolimus versus placebo in patients with advanced, non-functional, well-differentiated gastrointestinal or lung neuroendocrine tumours (RADIANT-4): a multicentre, randomised, double-blind, placebo-controlled, Phase III trial. Lancet Oncol. 18(10), 1411-1422 (2017).

- A comprehensive analysis of health-related quality of life (including the association with disease progression) in a clinical trial of metastatic cancer patients.

47 Huang IC, Willke RJ, Atkinson MJ, Lenderking WR, Frangakis C, Wu AW. US and UK versions of the EQ-5D preference weights: does choice of preference weights make a difference? Qual. Life Res. 16(6), 1065-1072 (2007).

48 Miller JD, Foley KA, Russell MW. Current challenges in health economic modeling of cancer therapies: a research inquiry. Am. Health Drug Benefits 7(3), 153-162 (2014). 